\title{
CORRECTIONS APPLIED TO THE CARRIER FREQUENCIES OF THE RADIO \\ STATIONS GBR AND RES ACCORDING TO THE USSR STATE \\ STANDARD OF TIME AND FREQUENCY
}

TABLE 1. Corrections Applied to the Carrier Frequencies of the Radio Stations GBR and RES According to the USSR State Standard of Time and Frequency

\begin{tabular}{|c|c|c|c|c|c|c|c|c|}
\hline \multicolumn{9}{|c|}{ February, 1971} \\
\hline date & $\begin{array}{l}\mathrm{GBR}, 16 \mathrm{kHz} \\
\text { referred to } \\
03 \mathrm{~h} \text { TU }\end{array}$ & $\begin{array}{l}\text { RES, } 100 \mathrm{kHz} \\
\text { referred to } \\
\text { to } 19 \mathrm{~h} \text { TU }\end{array}$ & date & $\begin{array}{l}\text { GBR, } 16 \mathrm{kHz} \\
\text { referred to } \\
03 \mathrm{~h} \mathrm{TU}\end{array}$ & $\begin{array}{l}\text { RES, } 100 \mathrm{kHz} \\
\text { referred to } \\
19 h \text { TU }\end{array}$ & date & $\begin{array}{l}\mathrm{GBR}, 16 \mathrm{kHz} \\
\text { referrdd to } \\
03 \mathrm{~h} \text { TI }\end{array}$ & $\begin{array}{l}\mathrm{RES}, 100 \mathrm{kHz} \\
\text { referred to } \\
\text { igh TU }\end{array}$ \\
\hline \multirow[t]{2}{*}{$\begin{array}{l}1 \\
2 \\
3 \\
4 \\
5 \\
6 \\
7 \\
8 \\
9\end{array}$} & $\begin{array}{l}-300,3 \cdot 10^{-10} \\
-300,3 \\
-299,8 \\
-300,3 \\
=300,1 \\
=299,8 \\
-299,9 \\
-299,4 \\
-300,5\end{array}$ & $\begin{array}{l}-299,4 \cdot 10^{-10} \\
-299,8 \\
-299,9 \\
-300,0 \\
-300,1 \\
-300,0 \\
-300,2 \\
-300,1 \\
-299,9\end{array}$ & $\begin{array}{l}10 \\
11 \\
12 \\
13 \\
14 \\
15 \\
16 \\
17 \\
18\end{array}$ & $\begin{array}{l}-299,7.10^{-10} \\
=300,0 \\
=300,2 \\
-299,6 \\
-300,3 \\
-299,5 \\
-300,0 \\
-300,0 \\
-300,1\end{array}$ & $\begin{array}{l}-300,0 \cdot 10^{-10} \\
-299,9 \\
-299,9 \\
-299,8 \\
-299,8 \\
-299,9 \\
-300,0 \\
-299,9 \\
-299,8\end{array}$ & $\begin{array}{l}19 \\
20 \\
21 \\
22 \\
23 \\
24 \\
25 \\
26 \\
27 \\
28\end{array}$ & $\begin{array}{l}-299,9 \cdot 10^{-10} \\
-300,1 \\
-299,6 \\
-300,0 \\
-300,0 \\
-299,9 \\
-299,6 \\
-300,0 \\
-300,1\end{array}$ & $\begin{array}{l}-299,9 \cdot 10^{-10} \\
-300,0 \\
-299,6 \\
-299,8 \\
-299,8 \\
-300,1 \\
-299,5 \\
-299,7 \\
-299,7 \\
-299,5\end{array}$ \\
\hline & & & & & & & $-300,0$ & $-299,9$ \\
\hline
\end{tabular}

Translated from Izmeritel'naya Tekhnika, No. 5, p. 77, May, 1971.

(C) 1971 Consultants Bureau, a division of Plenum Publishing Corporation, 227 West 17th Street, New York, N. Y. 100i1. All rights reserved. This article cannot be reproduced for any purpose whatsoever without permission of the publisher. A copy of this article is available from the publisher for $\$ 15.00$. 